\title{
State Estimation for Discrete-time Markovian Jumping Neural Networks with Mixed Mode-Dependent Delays
}

\author{
Yurong Liu, Zidong Wang* and Xiaohui Liu
}

\begin{abstract}
In this paper, we investigate the state estimation problem for a new class of discrete-time neural networks with Markovian jumping parameters as well as mode-dependent mixed time-delays. The parameters of the discrete-time neural networks are subject to the switching from one mode to another at different times according to a Markov chain, and the mixed time-delays consist of both discrete and distributed delays that are dependent on the Markovian jumping mode. New techniques are developed to deal with the mixed time-delays in the discrete-time setting, and a novel Lyapunov-Krasovskii functional is put forward to reflect the mode-dependent time-delays. Sufficient conditions are established in terms of linear matrix inequalities (LMIs) that guarantee the existence of the state estimators. We show that both the existence conditions and the explicit expression of the desired estimator can be characterized in terms of the solution to an LMI. A numerical example is exploited to show the usefulness of the derived LMI-based conditions.
\end{abstract}

\section{Keywords}

Discrete-time neural networks; Mixed time delays; Markovian jumping parameters; State estimator; Linear matrix inequality.

\section{INTRODUCTION}

It is now well known that many biological and artificial neural networks contain inherent time delays in signal transmission, which may cause oscillation and instability (see e.g. [1-3]). In recent years, a great number of papers have been published on various neural networks with time delays. The existence of equilibrium point, global asymptotic stability, global exponential stability, and the existence of periodic solutions have been intensively investigated, see e.g. [4-12]. For the dynamical behavior analysis of delayed neural networks, different types of time delays, such as constant delays, time-varying delays, and distributed delays, have been taken into account by using a variety of techniques that include linear matrix inequality (LMI) approach, Lyapunov functional method, $M$-matrix theory, topological degree theory, and techniques of inequality analysis. For example, in [10,13], the global asymptotic stability analysis problem has been dealt with for a class of neural networks with discrete and distributed time-delays by using an effective LMI approach.

In many applications, since the neuron states are not often fully available in the network outputs, the neuron state estimation problem becomes important. Thus, the state estimation problem for neural networks has recently drawn particular research interests, see e.g. [3,14-16]. For example, in [14], an adaptive state estimator has been described by using techniques of optimization theory, the calculus of variations and gradient descent dynamics. In [3], the neuron state estimation problem has been addressed for recurrent neural networks with time-varying delays, and an effective LMI approach has been developed to verify the stability of the estimation

This work was supported in part by the Biotechnology and Biological Sciences Research Council (BBSRC) of the UK under Grants BB/C506264/1 and 100/EGM17735, the Engineering and Physical Sciences Research Council (EPSRC) of the UK under Grants GR/S27658/01 and EP/C524586/1, an International Joint Project sponsored by the Royal Society of the UK, the Natural Science Foundation of Jiangsu Province of China under Grant BK2007075, the National Natural Science Foundation of China under Grant 60774073, and the Alexander von Humboldt Foundation of Germany.

Y. Liu is with the Department of Mathematics, Yangzhou University, Yangzhou 225002, P. R. China.

Z. Wang and X. Liu are with the Department of Information Systems and Computing, Brunel University, Uxbridge, Middlesex, UB8 3PH, United Kingdom.

Email addresses: liuyurong@gmail.com (Y. Liu), Zidong.Wang@brunel.ac.uk (Z. Wang).

${ }^{*}$ Corresponding author. 
error dynamics. Very recently, in [17], the robust state estimation problem has been studied for a class of uncertain neural networks with time-varying delay, and a sufficient condition has been presented to guarantee the existence of the desired state estimator for the uncertain delayed neural networks.

In practice, neural networks may be subject to network mode switching, which is governed by a Markovian chain [18]. In [19], the exponential stability has been studied for delayed recurrent neural networks with Markovian jumping parameters. In [20], the exponential synchronization problem has been investigated for a class of continuous-time complex networks with Markovian jump and mixed delays. On the other hand, discrete-time neural networks could be more suitable to model digitally transmitted signals in a dynamical way. However, to the best of the authors' knowledge, the state estimation problem for discrete-time Markovian jumping neural networks with or without mode-dependent mixed time-delays has not been adequately addressed in the literature yet, and the purpose of this paper is therefore to shorten such a gap.

In this paper, we deal with the state estimation problem for a new class of discrete-time neural networks with Markovian jumping parameters and mode-dependent mixed time-delays. By utilizing the Lyapunov functional method and some new techniques, we derive several delay-dependent sufficient conditions under which the estimation error dynamics is asymptotically stable. The criteria obtained in this paper are in the form of LMIs whose solution can be easily calculated by using the standard numerical software. Note that Markovian jumping parameters are introduced for discrete-time neural networks, and both the discrete and distributed delays are mode-dependent. A numerical example is presented to illustrate the usefulness of our results.

Notations: Throughout this paper, $\mathbb{R}^{n}$ and $\mathbb{R}^{n \times m}$ denote, respectively, the $n$ dimensional Euclidean space and the set of all $n \times m$ real matrices. The superscript " $T$ " denotes the transpose and the notation $X \geq Y$ (respectively, $X>Y$ ) where $X$ and $Y$ are symmetric matrices, means that $X-Y$ is positive semi-definite (respectively, positive definite); $I$ is the identity matrix with compatible dimension. $|\cdot|$ refers to the Euclidean vector norm. If $A$ is a symmetric matrix, $\lambda_{\min }(\cdot)$ and $\lambda_{\max }(\cdot)$ denote the minimum and the maximum eigenvalue, respectively. In symmetric block matrices, we use an asterisk "*" to represent a term that is induced by symmetry and $\operatorname{diag}\{\cdots\}$ stands for a block-diagonal matrix. $\mathbb{E}[x]$ and $\mathbb{E}[x \mid y]$ will, respectively, mean the expectation of $x$ and the expectation of $x$ conditional on $y$. Matrices, if their dimensions are not explicitly stated, are assumed to be compatible for algebraic operations.

\section{Problem formulation}

Let $r(k)(k \geq 0)$ be a Markov chain taking values in a finite state space $\mathcal{S}=\{1,2, \ldots, N\}$ with probability transition matrix $\boldsymbol{\Pi}=\left(\pi_{i j}\right)_{N \times N}$ given by

$$
\mathcal{P}\{r(k+1)=j \mid r(k)=i\}=\pi_{i j}, \quad \forall i, j \in \mathcal{S}
$$

where $\pi_{i j} \geq 0(i, j \in \mathcal{S})$ is the transition rate from $i$ to $j$ and $\sum_{j=1}^{N} \pi_{i j}=1, \forall i \in \mathcal{S}$.

Consider a discrete-time $n$-neuron neural network with $N$ modes described by the following dynamical equation:

$$
x(k+1)=D(r(k)) x(k)+A(r(k)) F(x(k))+B(r(k)) G\left(x\left(k-\tau_{1, r(k)}\right)\right)+C(r(k)) \sum_{v=1}^{\tau_{2, r(k)}} H(x(k-v))+J(r(k)),
$$

where $x(k)=\left(x_{1}(k), x_{2}(k), \ldots, x_{n}(k)\right)^{T}$ is the neural state vector; the constant matrices $D(r(k))=\operatorname{diag}\left\{d_{1}(r(k))\right.$, $\left.d_{2}(r(k)), \ldots, d_{n}(r(k))\right\}$ describe the rate with which the each neuron will reset its potential to the resting state in isolation when disconnected from the networks and external inputs; $A(r(k))=\left[a_{i j}(r(k))\right]_{n \times n}, B(r(k))=$ $\left[b_{i j}(r(k))\right]_{n \times n}$ and $C(r(k))=\left[c_{i j}(r(k))\right]_{n \times n}$ are, respectively, the connection weight matrix, the discretely delayed connection weight matrix and the distributively delayed connection weight matrix; $\tau_{1, r(k)}$ denotes discrete time delay while $\tau_{2, r(k)}$ characterizes distributed time delay, and both kinds of time delays are dependent on the system mode $r(k)$. In $(1), F(x(k))=\left(f_{1}\left(x_{1}(k)\right), f_{2}\left(x_{2}(k)\right), \ldots, f_{n}\left(x_{n}(k)\right)\right)^{T}, G\left(x\left(k-\tau_{1, r(k)}\right)\right)=\left(g_{1}\left(x_{1}(k-\right.\right.$ 
$\left.\left.\left.\tau_{1, r(k)}\right)\right), g_{2}\left(x_{2}\left(k-\tau_{1, r(k)}\right)\right), \ldots, g_{n}\left(x_{n}\left(k-\tau_{1, r(k)}\right)\right)\right)^{T}$ and $H(x(k))=\left(h_{1}\left(x_{1}(k)\right), h_{2}\left(x_{2}(k)\right), \ldots, h_{n}\left(x_{n}(k)\right)\right)^{T}$ are the nonlinear activation functions; and $J(r(k)))$ is constant vector representing the external bias on the neurons.

Remark 1: The discrete-time recurrent neural network (1) can be considered as a discrete analogue of the continuous-time recurrent neural network of the form:

$$
\frac{d x}{d t}=D(r(t)) x(t)+A(r(t)) F(x(t))+B(r(t)) G\left(x\left(t-\tau_{1, r(t)}\right)\right)+C(r(t)) \int_{t-\tau_{2, r(t)}}^{t} H(x(s)) d s+J(r(t)),
$$

where $r(t)$ corresponds to a Markov Chain. Note that the continuous-time neural networks without Markov chain have been extensively investigated; see, e.g. [13] and the references therein. However, to the best of authors' knowledge, discrete-time mode-dependent neural networks with or without mixed time delays, have received very little attention.

Throughout this paper, we make the following assumption.

Assumption 1 ( [13]) For the activation functions $F(\cdot), G(\cdot)$ and $H(\cdot)$, there exist constants $\lambda_{i}^{-}, \lambda_{i}^{+}, \sigma_{i}^{-}, \sigma_{i}^{+}$, $v_{i}^{-}$and $v_{i}^{+}$such that, for any $s_{1}, s_{2} \in \mathbb{R}, s_{1} \neq s_{2}, 1 \leq i \leq n$

$$
\lambda_{i}^{-} \leq \frac{f_{i}\left(s_{1}\right)-f_{i}\left(s_{2}\right)}{s_{1}-s_{2}} \leq \lambda_{i}^{+}, \quad \sigma_{i}^{-} \leq \frac{g_{i}\left(s_{1}\right)-g_{i}\left(s_{2}\right)}{s_{1}-s_{2}} \leq \sigma_{i}^{+}, \quad v_{i}^{-} \leq \frac{h_{i}\left(s_{1}\right)-h_{i}\left(s_{2}\right)}{s_{1}-s_{2}} \leq v_{i}^{+} .
$$

Remark 2: As pointed out in [13], the constants $\lambda_{i}^{-}, \lambda_{i}^{+}, \sigma_{i}^{-}, \sigma_{i}^{+}, v_{i}^{-}$and $v_{i}^{+}$in Assumption 1 are allowed to be positive, negative or zero. Hence, the resulting activation functions could be non-monotonic, and are more general than the usual sigmoid functions and the recently commonly used Lipschitz conditions. Such a description is very precise/tight in quantifying the lower and upper bounds of the activation functions, hence very helpful for using LMI-based approach to reduce the possible conservatism.

For neural networks, whether biological or artificial, it is usually difficult to get a complete access to their states and, in many applications, it consequently becomes necessary to estimate the states of neural networks based only on the output from the networks. In this paper, we assume that the output from the neural network (1) is of the form:

$$
y(k)=E(r(k)) x(k)+S(k, x(k))
$$

where $y(k)=\left(y_{1}(k), y_{2}(k), \ldots, y_{m}(k)\right)^{T}$ denotes the measurement output of the neural network, $E(i) \in \mathbb{R}^{m \times n}$ $(i \in \mathcal{S})$ is a constant matrix, and $S(k, x(k)) \in \mathbb{R}^{m}$ is a nonlinear disturbance dependent on the neuron state satisfying the following Lipschitz condition:

$$
|S(k, x)-S(k, y)| \leq|W(x-y)|, \forall k \in \mathbb{R}, x, y \in \mathbb{R}^{n}
$$

with $W$ being a known constant matrix of appropriate dimension.

Remark 3: Usually, the relationship $m<n$ holds, which means that only partial information (or a combination of the information) on the system states can be accessed via output measurements.

In order to track the state of system (1), we construct a full-order state estimator for (1):

$$
\begin{aligned}
\hat{x}(k+1)= & D(r(k)) \hat{x}(k)+A(r(k)) F(\hat{x}(k))+B(r(k)) G\left(\hat{x}\left(k-\tau_{1, r(k)}\right)\right)+C(r(k)) \sum_{v=1}^{\tau_{2, r(k)}} H(\hat{x}(k-v)) \\
& +J(r(k))-K(r(k))[y(k)-E(r(k)) \hat{x}(k)-S(k, \hat{x}(k))],
\end{aligned}
$$

where $\hat{x}(k)$ is the state estimate, and $K(i) \in \mathbb{R}^{n \times m}(i \in \mathcal{S})$ is the estimate gain matrix to be designed.

Let $\boldsymbol{\epsilon}(k)=\left(\epsilon_{1}(k), \epsilon_{2}(k), \ldots, \epsilon_{n}(k)\right)^{T} \triangleq \hat{x}(k)-x(k)$ be the state estimation error.

Definition 1: System (6) is said to be an asymptotic state estimator of neural network (1) if the estimation error satisfies

$$
\lim _{k \rightarrow+\infty} \mathbb{E}\left[|\boldsymbol{\epsilon}(k)|^{2}\right]=0 .
$$

This paper aims to design the state estimator of (1). By constructing new Lyapunov Krasovskii functional, we will derive the sufficient conditions under which system (6) becomes an asymptotic state estimator of neural network $(1)$, and the resulting gain matrices $K(i)(i \in \mathcal{S})$ will also be given explicitly. 


\section{MAIN RESUltS AND PROOFS}

The following lemmas are needed in deriving our results.

Lemma 1 ( [13]) Let $X, Y$ be any $n$-dimensional real vectors and $P$ be a $n \times n$ positive semi-definite matrix. Then, the following matrix inequality holds:

$$
2 X^{T} P Y \leq X^{T} P X+Y^{T} P Y .
$$

Lemma 2 ( [21]) Let $M \in \mathbb{R}^{n \times n}$ be a positive semi-definite matrix, $\mathbf{x}_{i} \in \mathbb{R}^{n}$ be a vector and $a_{i} \geq 0$ $(i=1,2, \ldots)$ be scalars. If the series concerned are convergent, then the following inequality holds:

$$
\left(\sum_{i=1}^{+\infty} a_{i} \mathbf{x}_{i}\right)^{T} M\left(\sum_{i=1}^{+\infty} a_{i} \mathbf{x}_{i}\right) \leq\left(\sum_{i=1}^{+\infty} a_{i}\right) \sum_{i=1}^{+\infty} a_{i} \mathbf{x}_{i}^{T} M \mathbf{x}_{i}
$$

Lemma 3: Suppose that $\mathcal{B}=\operatorname{diag}\left\{\beta_{1}, \beta_{2}, \ldots, \beta_{n}\right\} \geq 0$ is a diagonal matrix. Let $x=\left(x_{1}, x_{2}, \ldots, x_{n}\right)^{T} \in \mathbb{R}^{n}$, and $\mathcal{H}(x)=\left(\hbar_{1}\left(x_{1}\right), \hbar_{2}\left(x_{2}\right), \ldots, \hbar_{n}\left(x_{n}\right)\right)^{T}$ be a continuous nonlinear function satisfying

$$
l_{i}^{-} \leq \frac{\hbar_{i}(s)}{s} \leq l_{i}^{+}, s \neq 0, s \in \mathbb{R}, \quad i=1,2, \ldots, n
$$

with $l_{i}^{-}$and $l_{i}^{+}$being constant scalars. Then

$$
\left[\begin{array}{c}
x \\
\mathcal{H}(x)
\end{array}\right]^{T}\left[\begin{array}{cc}
\mathcal{B} L_{1} & -\mathcal{B} L_{2} \\
-\mathcal{B} L_{2} & \mathcal{B}
\end{array}\right]\left[\begin{array}{c}
x \\
\mathcal{H}(x)
\end{array}\right] \leq 0
$$

or

$$
x^{T} \mathcal{B} L_{1} x-2 x^{T} \mathcal{B} L_{2} \mathcal{H}(x)+\mathcal{H}^{T}(x) \mathcal{B H}(x) \leq 0
$$

where $L_{1}=\operatorname{diag}\left\{l_{1}^{+} l_{1}^{-}, l_{2}^{+} l_{2}^{-}, \ldots, l_{n}^{+} l_{n}^{-}\right\}$and $L_{2}=\operatorname{diag}\left\{\frac{l_{1}^{+}+l_{1}^{-}}{2}, \frac{l_{2}^{+}+l_{2}^{-}}{2}, \ldots, \frac{l_{n}^{+}+l_{n}^{-}}{2}\right\}$

Proof: Notice that (9) is equivalent to

$$
\left(\hbar_{i}\left(x_{i}\right)-l_{i}^{+} x_{i}\right)\left(\hbar_{i}\left(x_{i}\right)-l_{i}^{-} x_{i}\right) \leq 0
$$

or

$$
\left[\begin{array}{c}
x \\
\mathcal{H}(x)
\end{array}\right]^{T}\left[\begin{array}{cc}
l_{i}^{+} l_{i}^{-} e_{i} e_{i}^{T} & -\frac{l_{i}^{+}+l_{i}^{-}}{2} e_{i} e_{i}^{T} \\
-\frac{l_{i}^{+}+l_{i}^{-}}{2} e_{i} e_{i}^{T} & e_{i} e_{i}^{T}
\end{array}\right]\left[\begin{array}{c}
x \\
\mathcal{H}(x)
\end{array}\right] \leq 0, \quad i=1, \ldots, n,
$$

where $e_{k}$ denotes the unit column vector having "1" element on its $k$ th row and zeros elsewhere.

Since $\beta_{i} \geq 0$, it follows readily that

$$
\sum_{i=1}^{n} \beta_{i}\left[\begin{array}{c}
x \\
\mathcal{H}(x)
\end{array}\right]^{T}\left[\begin{array}{cc}
l_{i}^{+} l_{i}^{-} e_{i} e_{i}^{T} & -\frac{l_{i}^{+}+l_{i}^{-}}{2} e_{i} e_{i}^{T} \\
-\frac{l_{i}^{+}+l_{i}^{-}}{2} e_{i} e_{i}^{T} & e_{i} e_{i}^{T}
\end{array}\right]\left[\begin{array}{c}
x \\
\mathcal{H}(x)
\end{array}\right] \leq 0,
$$

namely

$$
\left[\begin{array}{c}
x \\
\mathcal{H}(x)
\end{array}\right]^{T}\left[\begin{array}{cc}
\mathcal{B} L_{1} & -\mathcal{B} L_{2} \\
-\mathcal{B} L_{2} & \mathcal{B}
\end{array}\right]\left[\begin{array}{c}
x \\
\mathcal{H}(x)
\end{array}\right] \leq 0 .
$$

This completes the proof of this lemma.

Lemma 4 ( [19]Schur Complement) Given constant matrices $\Omega_{1}, \Omega_{2}, \Omega_{3}$ where $\Omega_{1}=\Omega_{1}^{T}$ and $\Omega_{2}>0$, then

$$
\Omega_{1}+\Omega_{3}^{T} \Omega_{2}^{-1} \Omega_{3}<0
$$

if only if

$$
\left[\begin{array}{cc}
\Omega_{1} & \Omega_{3}^{T} \\
\Omega_{3} & -\Omega_{2}
\end{array}\right]<0
$$


In what follows, for presentation convenience, we denote

$$
\begin{aligned}
& \bar{\tau}_{1}=\max \left\{\tau_{1, i} \mid i \in \mathcal{S}\right\}, \bar{\tau}_{2}=\max \left\{\tau_{2, i} \mid i \in \mathcal{S}\right\}, \tau=\max \left\{\bar{\tau}_{1}, \bar{\tau}_{2}\right\}, \\
& \underline{\tau}_{1}=\min \left\{\tau_{1, i} \mid i \in \mathcal{S}\right\}, \underline{\tau}_{2}=\min \left\{\tau_{2, i} \mid i \in \mathcal{S}\right\}, \underline{\pi}=\min \left\{\pi_{i i} \mid i \in \mathcal{S}\right\}, \\
& \Lambda_{1}=\operatorname{diag}\left\{\lambda_{1}^{+} \lambda_{1}^{-}, \lambda_{2}^{+} \lambda_{2}^{-}, \ldots, \lambda_{n}^{+} \lambda_{n}^{-}\right\}, \Lambda_{2}=\operatorname{diag}\left\{\frac{\lambda_{1}^{+}+\lambda_{1}^{-}}{2}, \frac{\lambda_{2}^{+}+\lambda_{2}^{-}}{2}, \ldots, \frac{\lambda_{n}^{+}+\lambda_{n}^{-}}{2}\right\}, \\
& \Sigma_{1}=\operatorname{diag}\left\{\sigma_{1}^{+} \sigma_{1}^{-}, \sigma_{2}^{+} \sigma_{2}^{-}, \ldots, \sigma_{n}^{+} \sigma_{n}^{-}\right\}, \Sigma_{2}=\operatorname{diag}\left\{\frac{\sigma_{1}^{+}+\sigma_{1}^{-}}{2}, \frac{\sigma_{2}^{+}+\sigma_{2}^{-}}{2}, \ldots, \frac{\sigma_{n}^{+}+\sigma_{n}^{-}}{2}\right\}, \\
& \Upsilon_{1}=\operatorname{diag}\left\{v_{1}^{+} v_{1}^{-}, v_{2}^{+} v_{2}^{-}, \ldots, v_{n}^{+} v_{n}^{-}\right\}, \Upsilon_{2}=\operatorname{diag}\left\{\frac{v_{1}^{+}+v_{1}^{-}}{2}, \frac{v_{2}^{+}+v_{2}^{-}}{2}, \ldots, \frac{v_{n}^{+}+v_{n}^{-}}{2}\right\} .
\end{aligned}
$$

Theorem 1: Suppose that Assumption 1 and condition (5) hold and Let $K(i)(i \in \mathcal{S})$ be known constant matrices. Then system (6) becomes an asymptotic state estimator of neural network (1) if there exist a set of scalar constants $\vartheta_{i}>0(i \in \mathcal{S})$, a set of matrices $P_{i}>0(i \in \mathcal{S})$, two matrices $Q>0$ and $R>0$, and three sets of diagonal matrices $\Omega_{i}>0, \Theta_{i}>0$ and $\Delta_{i}>0(i \in \mathcal{S})$ such that the following LMIs hold:

$$
\Phi_{i} \triangleq\left[\begin{array}{cccccccc}
\Xi_{i} & \Omega_{i} \Lambda_{2} & \Theta_{i} \Sigma_{2} & 0 & \Delta_{i} \Upsilon_{2} & 0 & 0 & \bar{D}^{T}(i) \bar{P}_{i} \\
\Omega_{i} \Lambda_{2} & -\Omega_{i} & 0 & 0 & 0 & 0 & 0 & A^{T}(i) \bar{P}_{i} \\
\Theta_{i} \Sigma_{2} & 0 & \kappa_{0} Q-\Theta_{i} & 0 & 0 & 0 & 0 & 0 \\
0 & 0 & 0 & -Q & 0 & 0 & 0 & B^{T}(i) \bar{P}_{i} \\
\Delta_{i} \Upsilon_{2} & 0 & 0 & 0 & \kappa_{i} R-\Delta_{i} & 0 & 0 & 0 \\
0 & 0 & 0 & 0 & 0 & \frac{1}{\tau_{2, i}} R & 0 & C^{T}(i) \bar{P}_{i} \\
0 & 0 & 0 & 0 & 0 & 0 & -\vartheta_{i} I & \bar{P}_{i} \\
\bar{P}_{i} \bar{D}(i) & \bar{P}_{i} A(i) & 0 & \bar{P}_{i} B(i) & 0 & \bar{P}_{i} C(i) & \bar{P}_{i} & -\bar{P}_{i}
\end{array}\right]<0, \quad(i \in \mathcal{S})
$$

where

$$
\begin{aligned}
& \bar{P}_{i}=\sum_{j=1}^{N} \pi_{i j} P_{j}, \bar{D}(i)=D(i)+K(i) E(i), \\
& \Xi_{i}=-P_{i}-\Omega_{i} \Lambda_{1}-\Theta_{i} \Sigma_{1}-\Delta_{i} \Upsilon_{1}+\vartheta_{i} W^{T} W \\
& \kappa_{0}=(1-\underline{\pi})\left(\bar{\tau}_{1}-\underline{\tau}_{1}\right)+1, \\
& \kappa_{i}=\tau_{2, i}+\left(1-\pi_{i i}\right)\left(\bar{\tau}_{2}-\underline{\tau}_{2}\right)+\frac{1}{2}(1-\underline{\pi})\left(\bar{\tau}_{2}-\underline{\tau}_{2}\right)\left(\bar{\tau}_{2}+\underline{\tau}_{2}-1\right) .
\end{aligned}
$$

Proof: Denote

$$
\begin{aligned}
& \tilde{F}(\boldsymbol{\epsilon}(k))=\left[\tilde{f}_{1}\left(\epsilon_{1}(k)\right), \tilde{f}_{2}\left(\epsilon_{1}(k)\right), \ldots, \tilde{f}_{n}\left(\epsilon_{n}(k)\right)\right]^{T} \triangleq F(\hat{x}(k))-F(x(k)), \\
& \tilde{G}(\boldsymbol{\epsilon}(k))=\left[\tilde{g}_{1}\left(\epsilon_{1}(k)\right), \tilde{g}_{2}\left(\epsilon_{1}(k)\right), \ldots, \tilde{g}_{n}\left(\epsilon_{n}(k)\right)\right]^{T} \triangleq G(\hat{x}(k))-G(x(k)), \\
& \tilde{H}(\boldsymbol{\epsilon}(k))=\left[\tilde{h}_{1}\left(\epsilon_{1}(k)\right), \tilde{h}_{2}\left(\epsilon_{1}(k)\right), \ldots, \tilde{h}_{n}\left(\epsilon_{n}(k)\right)\right]^{T} \triangleq H(\hat{x}(k))-H(x(k)), \\
& \tilde{S}(k, \boldsymbol{\epsilon}(k)) \triangleq S(k, \hat{x}(k))-S(k, x(k)) .
\end{aligned}
$$

It should be pointed out here that $\tilde{F}(\boldsymbol{\epsilon}(k)), \tilde{G}(\boldsymbol{\epsilon}(k)), \tilde{H}(\boldsymbol{\epsilon}(k))$ and $\tilde{S}(k, \boldsymbol{\epsilon}(k))$ are all dependent on $x(k)$ or $\hat{x}(k)$, as well as $\boldsymbol{\epsilon}(k)$. However, in order to avoid cumbersome notations, we shall use simpler symbols. For instance, we use $\tilde{F}(\boldsymbol{\epsilon}(k))$ instead of $\tilde{F}(\boldsymbol{\epsilon}(k), x(k))$ and so on.

With the above notations and from Eqs. (1) and (6), we obtain the dynamics of estimation error governed by

$$
\begin{aligned}
\boldsymbol{\epsilon}(k+1)= & \bar{D}(r(k)) \boldsymbol{\epsilon}(k)+A(r(k)) \tilde{F}(\boldsymbol{\epsilon}(k))+B(r(k)) \tilde{G}\left(\boldsymbol{\epsilon}\left(k-\tau_{1, r(k)}\right)\right) \\
& +C(r(k)) \sum_{v=1}^{\tau_{2, r(k)}} \tilde{H}(\boldsymbol{\epsilon}(k-v))+K(r(k)) \tilde{S}(k, \boldsymbol{\epsilon}(k)) .
\end{aligned}
$$


For notation convenience, in the sequel, we denote

$$
\begin{aligned}
& \boldsymbol{\epsilon}_{k}=\left[\boldsymbol{\epsilon}^{T}(k), \boldsymbol{\epsilon}^{T}(k-1), \ldots, \boldsymbol{\epsilon}^{T}(k-\tau)\right]^{T}, \\
& \xi(k, i)=\left[\begin{array}{lllllll}
\boldsymbol{\epsilon}^{T}(k) & \tilde{F}^{T}(\boldsymbol{\epsilon}(k)) & \tilde{G}^{T}(\boldsymbol{\epsilon}(k)) & \tilde{G}^{T}\left(\boldsymbol{\epsilon}\left(k-\tau_{1, i}\right)\right) & \tilde{H}^{T}(\boldsymbol{\epsilon}(k)) & \sum_{v=1}^{\tau_{2, i}} \tilde{H}^{T}(\boldsymbol{\epsilon}(k-v)) & \tilde{S}^{T}(k, \boldsymbol{\epsilon}(k))
\end{array}\right]^{T}, \\
& \mathcal{Z}(i)=\left[\begin{array}{lllllll}
\bar{D}(i) & A(i) & 0 & B(i) & 0 & C(i) & I
\end{array}\right] .
\end{aligned}
$$

Now, in order to ensure that (6) is the state estimator, we just need to show that the estimation error system (20) is asymptotically stable in the mean square, i.e., $\lim _{k \rightarrow \infty} \mathbb{E}\left[|\boldsymbol{\epsilon}(k)|^{2}\right]=0$. To this end, we construct the following Lyapunov-Krasovskii functional $V\left(\mathbf{x}_{k}, k, r(k)\right)$ by

$$
V\left(\boldsymbol{\epsilon}_{k}, k, r(k)\right)=V_{1}\left(\boldsymbol{\epsilon}_{k}, k, r(k)\right)+V_{2}\left(\boldsymbol{\epsilon}_{k}, k, r(k)\right)+V_{3}\left(\boldsymbol{\epsilon}_{k}, k, r(k)\right)+V_{4}\left(\boldsymbol{\epsilon}_{k}, k, r(k)\right)+V_{5}\left(\boldsymbol{\epsilon}_{k}, k, r(k)\right)
$$

where

$$
\begin{aligned}
& V_{1}\left(\boldsymbol{\epsilon}_{k}, k, r(k)\right)=\boldsymbol{\epsilon}^{T}(k) P_{r(k)} \boldsymbol{\epsilon}(k), \\
& V_{2}\left(\boldsymbol{\epsilon}_{k}, k, r(k)\right)=\sum_{v=k-\tau_{1, r(k)}}^{k-1} \tilde{G}^{T}(\boldsymbol{\epsilon}(v)) Q \tilde{G}(\boldsymbol{\epsilon}(v)), \\
& V_{3}\left(\boldsymbol{\epsilon}_{k}, k, r(k)\right)=\sum_{\iota=\underline{\tau}_{1}}^{\bar{\tau}_{1}-1} \sum_{v=k-\iota}^{k-1} \tilde{G}^{T}(\boldsymbol{\epsilon}(v)) \bar{Q} \tilde{G}(\boldsymbol{\epsilon}(v)), \\
& V_{4}\left(\boldsymbol{\epsilon}_{k}, k, r(k)\right)=\sum_{\iota=1}^{\tau_{2, r(k)}} \sum_{v=k-\iota}^{k-1} \tilde{H}^{T}(\boldsymbol{\epsilon}(v)) R \tilde{H}(\boldsymbol{\epsilon}(v)), \\
& V_{5}\left(\boldsymbol{\epsilon}_{k}, k, r(k)\right)=\sum_{s=\underline{\tau}_{2}+1}^{\bar{\tau}_{2}} \sum_{\iota=1}^{s-1} \sum_{v=k-\iota}^{k-1} \tilde{H}^{T}(\boldsymbol{\epsilon}(v)) \bar{R} \tilde{H}(\boldsymbol{\epsilon}(v))
\end{aligned}
$$

with $\bar{Q}=(1-\underline{\pi}) Q$ and $\bar{R}=(1-\underline{\pi}) R$.

For $i \in \mathcal{S}$, we have

$$
\begin{aligned}
& \mathbb{E}\left[V_{1}\left(\boldsymbol{\epsilon}_{k+1}, k+1, r(k+1)\right) \mid \boldsymbol{\epsilon}_{k}, r(k)=i\right]-V_{1}\left(\boldsymbol{\epsilon}_{k}, k, i\right) \\
= & \xi^{T}(k, i) \mathcal{Z}^{T}(i) \bar{P}_{i} \mathcal{Z}(i) \xi(k, i)-\boldsymbol{\epsilon}^{T}(k) P_{i} \boldsymbol{\epsilon}(k) .
\end{aligned}
$$

$$
\begin{aligned}
& \mathbb{E}\left[V_{2}\left(\boldsymbol{\epsilon}_{k+1}, k+1, r(k+1)\right) \mid \boldsymbol{\epsilon}_{k}, r(k)=i\right]-V_{2}\left(\boldsymbol{\epsilon}_{k}, k, i\right) \\
& =\sum_{j=1}^{N} \pi_{i j} \sum_{v=k-\tau_{1, j}+1}^{k} \tilde{G}^{T}(\boldsymbol{\epsilon}(v)) Q \tilde{G}(\boldsymbol{\epsilon}(v))-\sum_{v=k-\tau_{1, i}}^{k-1} \tilde{G}^{T}(\boldsymbol{\epsilon}(v)) Q \tilde{G}(\boldsymbol{\epsilon}(v)) \\
& =\pi_{i i}\left[\sum_{v=k-\tau_{1, i}+1}^{k}-\sum_{v=k-\tau_{1, i}}^{k-1}\right] \tilde{G}^{T}(\boldsymbol{\epsilon}(v)) Q \tilde{G}(\boldsymbol{\epsilon}(v))+\sum_{j \neq i} \pi_{i j}\left[\sum_{v=k-\tau_{1, j}+1}^{k}-\sum_{v=k-\tau_{1, i}}^{k-1}\right] \tilde{G}^{T}(\boldsymbol{\epsilon}(k)) Q \tilde{G}(\boldsymbol{\epsilon}(k)) \\
& \leq \tilde{G}^{T}(\boldsymbol{\epsilon}(k)) Q \tilde{G}(\boldsymbol{\epsilon}(k))-\tilde{G}^{T}\left(\boldsymbol{\epsilon}\left(k-\tau_{1, i}\right)\right) Q \tilde{G}\left(\boldsymbol{\epsilon}\left(k-\tau_{1, i}\right)\right)+(1-\underline{\pi}) \sum_{v=k-\bar{\tau}_{1}+1}^{k-\underline{\tau}_{1}} \tilde{G}^{T}(\boldsymbol{\epsilon}(v)) Q \tilde{G}(\boldsymbol{\epsilon}(v)), \\
& \mathbb{E}\left[V_{3}\left(\boldsymbol{\epsilon}_{k+1}, k+1, r(k+1)\right) \mid \boldsymbol{\epsilon}_{k}, r(k)=i\right]-V_{3}\left(\boldsymbol{\epsilon}_{k}, k, i\right) \\
& =\sum_{\iota=\underline{\tau}_{1}}^{\bar{\tau}_{1}-1}\left(\tilde{G}^{T}(\boldsymbol{\epsilon}(k)) \bar{Q} \tilde{G}(\boldsymbol{\epsilon}(k))-\tilde{G}^{T}(\boldsymbol{\epsilon}(k-\iota)) \bar{Q} \tilde{G}(\boldsymbol{\epsilon}(k-\iota))\right) \\
& =(1-\underline{\pi})\left(\bar{\tau}_{1}-\underline{\tau}_{1}\right) \tilde{G}^{T}(\boldsymbol{\epsilon}(k)) Q \tilde{G}(\boldsymbol{\epsilon}(k))-(1-\underline{\pi}) \sum_{v=k-\bar{\tau}_{1}+1}^{k-\underline{\tau}_{1}} \tilde{G}^{T}(\boldsymbol{\epsilon}(v)) Q \tilde{G}(\boldsymbol{\epsilon}(v)),
\end{aligned}
$$




$$
\begin{aligned}
& \mathbb{E}\left[V_{4}\left(\boldsymbol{\epsilon}_{k+1}, k+1, r(k+1)\right) \mid \boldsymbol{\epsilon}_{k}, r(k)=i\right]-V_{4}\left(\boldsymbol{\epsilon}_{k}, k, i\right) \\
& =\sum_{j=1}^{N} \pi_{i j} \sum_{\iota=1}^{\tau_{2, j}} \sum_{v=k-\iota+1}^{k} \tilde{H}^{T}(\boldsymbol{\epsilon}(v)) R \tilde{H}(\boldsymbol{\epsilon}(v))-\sum_{\iota=1}^{\tau_{2, i}} \sum_{v=k-\iota}^{k-1} \tilde{H}^{T}(\boldsymbol{\epsilon}(v)) R \tilde{H}(\boldsymbol{\epsilon}(v)) \\
& =\pi_{i i} \sum_{\iota=1}^{\tau_{2, i}}\left[\tilde{H}^{T}(\boldsymbol{\epsilon}(k)) R \tilde{H}(\boldsymbol{\epsilon}(k))-\tilde{H}^{T}(\boldsymbol{\epsilon}(k-\iota)) R \tilde{H}(\boldsymbol{\epsilon}(k-\iota))\right] \\
& +\sum_{j \neq i} \pi_{i j}\left[\sum_{\iota=1}^{\tau_{2, j}} \sum_{v=k-\iota+1}^{k}-\sum_{\iota=1}^{\tau_{2, i}} \sum_{v=k-\iota}^{k-1}\right] \tilde{H}^{T}(\boldsymbol{\epsilon}(v)) R \tilde{H}(\boldsymbol{\epsilon}(v)) \\
& =\pi_{i i} \sum_{\iota=1}^{\tau_{2, i}}\left[\tilde{H}^{T}(\boldsymbol{\epsilon}(k)) R \tilde{H}(\boldsymbol{\epsilon}(k))-\tilde{H}^{T}(\boldsymbol{\epsilon}(k-\iota)) R \tilde{H}(\boldsymbol{\epsilon}(k-\iota))\right] \\
& +\sum_{j \neq i} \pi_{i j}\left[\sum_{\iota=1}^{\tau_{2, i}} \sum_{v=k-\iota+1}^{k}-\sum_{\iota=1}^{\tau_{2, i}} \sum_{v=k-\iota}^{k-1}\right] \tilde{H}^{T}(\boldsymbol{\epsilon}(v)) R \tilde{H}(\boldsymbol{\epsilon}(v)) \\
& +\sum_{j \neq i} \pi_{i j}\left[\sum_{\iota=1}^{\tau_{2, j}} \sum_{v=k-\iota+1}^{k}-\sum_{\iota=1}^{\tau_{2, i}} \sum_{v=k-\iota+1}^{k}\right] \tilde{H}^{T}(\boldsymbol{\epsilon}(v)) R \tilde{H}(\boldsymbol{\epsilon}(v)) \\
& \leq \sum_{\iota=1}^{\tau_{2, i}}\left(\tilde{H}^{T}(\boldsymbol{\epsilon}(k)) R \tilde{H}(\boldsymbol{\epsilon}(k))-\tilde{H}^{T}(\boldsymbol{\epsilon}(k-\iota)) R \tilde{H}(\boldsymbol{\epsilon}(k-\iota))\right) \\
& +\sum_{j \neq i} \pi_{i j}\left[\sum_{\iota=\underline{\tau}_{2}+1}^{\bar{\tau}_{2}} \sum_{v=k-\iota+1}^{k} \tilde{H}^{T}(\boldsymbol{\epsilon}(v)) R \tilde{H}(\boldsymbol{\epsilon}(v))\right] \\
& =\tau_{2, i} \tilde{H}^{T}(\boldsymbol{\epsilon}(k)) R \tilde{H}(\boldsymbol{\epsilon}(k))-\sum_{\iota=1}^{\tau_{2, i}} \tilde{H}^{T}(x(k-\iota)) R \tilde{H}(x(k-\iota)) \\
& +\left(1-\pi_{i i}\right) \sum_{\iota=\underline{\tau}_{2}+1}^{\bar{\tau}_{2}} \sum_{v=k-\iota+1}^{k} \tilde{H}^{T}(\boldsymbol{\epsilon}(v)) R \tilde{H}(\boldsymbol{\epsilon}(v)) \\
& \leq\left(\tau_{2, i}+\left(1-\pi_{i i}\right)\left(\bar{\tau}_{2}-\underline{\tau}_{2}\right)\right) \tilde{H}^{T}(\boldsymbol{\epsilon}(k)) R \tilde{H}(\boldsymbol{\epsilon}(k))-\sum_{v=1}^{\tau_{2, i}} \tilde{H}^{T}(\boldsymbol{\epsilon}(k-v)) R \tilde{H}(\boldsymbol{\epsilon}(k-v)) \\
& +(1-\underline{\pi}) \sum_{\iota=\underline{\tau}_{2}+1}^{\bar{\tau}_{2}} \sum_{v=k-\iota+1}^{k-1} \tilde{H}^{T}(\boldsymbol{\epsilon}(v)) R \tilde{H}(\boldsymbol{\epsilon}(v)),
\end{aligned}
$$

and

$$
\begin{aligned}
& \mathbb{E}\left[V_{5}\left(\boldsymbol{\epsilon}_{k+1}, k+1, r(k+1)\right) \mid \boldsymbol{\epsilon}_{k}, r(k)=i\right]-V_{5}\left(\boldsymbol{\epsilon}_{k}, k, i\right) \\
= & \sum_{s=\underline{\tau}_{2}+1}^{\bar{\tau}_{2}} \sum_{\iota=1}^{s-1}\left[\tilde{H}^{T}(\boldsymbol{\epsilon}(k)) \bar{R} \tilde{H}(\boldsymbol{\epsilon}(k))-\tilde{H}^{T}(\boldsymbol{\epsilon}(k-\iota)) \bar{R} \tilde{H}(\boldsymbol{\epsilon}(k-\iota))\right] \\
= & (1-\underline{\pi})\left[\frac{1}{2}\left(\bar{\tau}_{2}-\underline{\tau}_{2}\right)\left(\bar{\tau}_{2}+\underline{\tau}_{2}-1\right) \tilde{H}^{T}(\boldsymbol{\epsilon}(k)) R \tilde{H}(\boldsymbol{\epsilon}(k))-\sum_{\iota=\underline{\tau}_{2}+1}^{\bar{\tau}_{2}} \sum_{v=k-\iota+1}^{k-1} \tilde{H}^{T}(\boldsymbol{\epsilon}(v)) R \tilde{H}(\boldsymbol{\epsilon}(v))\right] .
\end{aligned}
$$


From (27)-(31), it follows readily that

$$
\begin{aligned}
& \mathbb{E}\left[V\left(\boldsymbol{\epsilon}_{k+1}, k+1, r(k+1)\right) \mid \boldsymbol{\epsilon}_{k}, r(k)=i\right]-V\left(\boldsymbol{\epsilon}_{k}, k, i\right) \\
\leq \quad & \xi^{T}(k, i) \mathcal{Z}^{T}(i) \bar{P}_{i} \mathcal{Z}(i) \xi(k, i)-\boldsymbol{\epsilon}^{T}(k) P_{i} \boldsymbol{\epsilon}(k)+\kappa_{0} \tilde{G}^{T}(\boldsymbol{\epsilon}(k)) Q \tilde{G}(\boldsymbol{\epsilon}(k)) \\
& -\tilde{G}^{T}\left(\boldsymbol{\epsilon}\left(k-\tau_{1, i}\right)\right) Q \tilde{G}\left(\boldsymbol{\epsilon}\left(k-\tau_{1, i}\right)\right)+\kappa_{i} \tilde{H}^{T}(\boldsymbol{\epsilon}(k)) R \tilde{H}(\boldsymbol{\epsilon}(k))-\sum_{v=1}^{\tau_{2, i}} \tilde{H}^{T}(\boldsymbol{\epsilon}(k-v)) R \tilde{H}(\boldsymbol{\epsilon}(k-v)),
\end{aligned}
$$

where $\kappa_{0}$ and $\kappa_{i}$ are defined in (14) and (15), respectively.

In terms of Lemma 2, one knows

$$
-\sum_{v=1}^{\tau_{2, i}} \tilde{H}^{T}(\boldsymbol{\epsilon}(k-v)) R \tilde{H}(\boldsymbol{\epsilon}(k-v)) \leq-\frac{1}{\tau_{2, i}}\left[\sum_{v=1}^{\tau_{2, i}} \tilde{H}(\boldsymbol{\epsilon}(k-v))\right]^{T} R \sum_{v=1}^{\tau_{2, i}} \tilde{H}(\boldsymbol{\epsilon}(k-v)) .
$$

From Assumption 1 and (16)-(19), it is not difficult to see that for any $s_{1}, s_{2} \in \mathbb{R}, s_{1} \neq s_{2}, 1 \leq i \leq n$

$$
\lambda_{i}^{-} \leq \frac{\tilde{f}_{i}\left(s_{1}\right)-\tilde{f}_{i}\left(s_{2}\right)}{s_{1}-s_{2}} \leq \lambda_{i}^{+}, \quad \sigma_{i}^{-} \leq \frac{\tilde{g}_{i}\left(s_{1}\right)-\tilde{g}_{i}\left(s_{2}\right)}{s_{1}-s_{2}} \leq \sigma_{i}^{+}, \quad v_{i}^{-} \leq \frac{\tilde{h}_{i}\left(s_{1}\right)-\tilde{h}_{i}\left(s_{2}\right)}{s_{1}-s_{2}} \leq v_{i}^{+}
$$

and

$$
\tilde{f}_{i}(0)=\tilde{g}_{i}(0)=\tilde{h}_{i}(0)=0 .
$$

Consequently, it follows that for any $s \in \mathbb{R}, s \neq 0,1 \leq i \leq n$

$$
\lambda_{i}^{-} \leq \frac{\tilde{f}_{i}(s)}{s} \leq \lambda_{i}^{+}, \quad \sigma_{i}^{-} \leq \frac{\tilde{g}_{i}(s)}{s} \leq \sigma_{i}^{+}, \quad v_{i}^{-} \leq \frac{\tilde{h}_{i}(s)}{s} \leq v_{i}^{+} .
$$

Also, from Lemma 3, it implies that

$$
\begin{aligned}
& \boldsymbol{\epsilon}^{T}(k) \Omega_{i} \Lambda_{1} \boldsymbol{\epsilon}(k)-2 \boldsymbol{\epsilon}^{T}(k) \Omega_{i} \Lambda_{2} \tilde{F}(\boldsymbol{\epsilon}(k))+\tilde{F}^{T}(\boldsymbol{\epsilon}(k)) \Omega_{i} \tilde{F}(\boldsymbol{\epsilon}(k)) \leq 0, \\
& \boldsymbol{\epsilon}^{T}(k) \Theta_{i} \Sigma_{1} \boldsymbol{\epsilon}(k)-2 \boldsymbol{\epsilon}^{T}(k) \Theta_{i} \Sigma_{2} \tilde{G}(\boldsymbol{\epsilon}(k))+\tilde{G}^{T}(\boldsymbol{\epsilon}(k)) \Theta_{i} \tilde{G}(\boldsymbol{\epsilon}(k)) \leq 0, \\
& \boldsymbol{\epsilon}^{T}(k) \Delta_{i} \Upsilon_{1} \boldsymbol{\epsilon}(k)-2 \boldsymbol{\epsilon}^{T}(k) \Delta_{i} \Upsilon_{2} \tilde{H}(\boldsymbol{\epsilon}(k))+\tilde{H}^{T}(\boldsymbol{\epsilon}(k)) \Delta_{i} \tilde{H}(\boldsymbol{\epsilon}(k)) \leq 0 .
\end{aligned}
$$

Moreover, from (5) and (19), it follows that

$$
|\tilde{S}(k, x)-\tilde{S}(k, y)| \leq|W(x-y)|, \forall x, y \in \mathbb{R}^{n},
$$

and $\tilde{S}(t, 0)=0$, which implies that

$$
\vartheta_{i} \tilde{S}^{T}(k, \boldsymbol{\epsilon}(k)) \tilde{S}(k, \boldsymbol{\epsilon}(k))-\vartheta_{i} \boldsymbol{\epsilon}^{T}(k) W^{T} W \boldsymbol{\epsilon}(k) \leq 0 .
$$

Combination of (33)-(38) with (32) results in

$$
\begin{aligned}
& \mathbb{E}\left[V\left(\boldsymbol{\epsilon}_{k+1}, k+1, r(k+1)\right) \mid \boldsymbol{\epsilon}_{k}, r(k)=i\right]-V\left(\boldsymbol{\epsilon}_{k}, k, i\right) \\
\leq \quad & \xi^{T}(k, i) \mathcal{Z}^{T}(i) \bar{P}_{i} \mathcal{Z}(i) \xi(k, i)-\boldsymbol{\epsilon}^{T}(k) P_{i} \boldsymbol{\epsilon}(k)+\kappa_{0} \tilde{G}^{T}(\boldsymbol{\epsilon}(k)) Q \tilde{G}(\boldsymbol{\epsilon}(k))-\tilde{G}^{T}\left(\boldsymbol{\epsilon}\left(k-\tau_{1, i}\right)\right) Q \tilde{G}\left(\boldsymbol{\epsilon}\left(k-\tau_{1, i}\right)\right) \\
& +\kappa_{i} \tilde{H}^{T}(\boldsymbol{\epsilon}(k)) R \tilde{H}(\boldsymbol{\epsilon}(k))-\frac{1}{\tau_{2, i}}\left[\sum_{v=1}^{\tau_{2, i}} \tilde{H}(\boldsymbol{\epsilon}(k-v))\right]^{T} R \sum_{v=1}^{\tau_{2, i}} \tilde{H}(\boldsymbol{\epsilon}(k-v))-\left(\boldsymbol{\epsilon}^{T}(k) \Omega_{i} \Lambda_{1} \boldsymbol{\epsilon}(k)\right. \\
& \left.-2 \boldsymbol{\epsilon}^{T}(k) \Omega_{i} \Lambda_{2} \tilde{F}(\boldsymbol{\epsilon}(k))+\tilde{F}^{T}(\boldsymbol{\epsilon}(k)) \Omega_{i} \tilde{F}(\boldsymbol{\epsilon}(k))\right)-\left(\boldsymbol{\epsilon}^{T}(k) \Theta_{i} \Sigma_{1} \boldsymbol{\epsilon}(k)-2 \boldsymbol{\epsilon}^{T}(k) \Theta_{i} \Sigma_{2} \tilde{G}(\boldsymbol{\epsilon}(k))\right. \\
& \left.+\tilde{G}^{T}(\boldsymbol{\epsilon}(k)) \Theta_{i} \tilde{G}(\boldsymbol{\epsilon}(k))\right)-\left(\boldsymbol{\epsilon}^{T}(k) \Delta_{i} \Upsilon_{1} \boldsymbol{\epsilon}(k)-2 \boldsymbol{\epsilon}^{T}(k) \Delta_{i} \Upsilon_{2} \tilde{H}(\boldsymbol{\epsilon}(k))+\tilde{H}^{T}(\boldsymbol{\epsilon}(k)) \Delta_{i} \tilde{H}(\boldsymbol{\epsilon}(k))\right) \\
& -\left(\vartheta_{i} \tilde{S}^{T}(k, \boldsymbol{\epsilon}(k)) \tilde{S}(k, \boldsymbol{\epsilon}(k))-\vartheta_{i} \boldsymbol{\epsilon}^{T}(k) W^{T} W \boldsymbol{\epsilon}(k)\right) \\
= & \xi^{T}(k, i) \tilde{\Psi}_{i} \xi(k, i),
\end{aligned}
$$


where $\tilde{\Psi}_{i}=\Psi_{i}+\mathcal{Z}^{T}(i) \bar{P}_{i} \mathcal{Z}(i)$ with

$$
\Psi_{i}=\left[\begin{array}{ccccccc}
\Xi_{i} & \Omega_{i} \Lambda_{2} & \Theta_{i} \Sigma_{2} & 0 & \Delta_{i} \Upsilon_{2} & 0 & 0 \\
\Omega_{i} \Lambda_{2} & -\Omega_{i} & 0 & 0 & 0 & 0 & 0 \\
\Theta_{i} \Sigma_{2} & 0 & \kappa_{0} Q-\Theta_{i} & 0 & 0 & 0 & 0 \\
0 & 0 & 0 & -Q & 0 & 0 & 0 \\
\Delta_{i} \Upsilon_{2} & 0 & 0 & 0 & \kappa_{i} R-\Delta_{i} & 0 & 0 \\
0 & 0 & 0 & 0 & 0 & \frac{1}{\tau_{2, i}} R & 0 \\
0 & 0 & 0 & 0 & 0 & 0 & -\vartheta_{i} I
\end{array}\right]
$$

and $\Xi_{i}$ defined in (13).

By applying Lemma 4 to $(11)$, we can deduce that $\tilde{\Psi}_{i}<0,(i \in \mathcal{S})$. Let $\alpha_{0}=\max _{i \in \mathcal{S}}\left\{\lambda_{\max }\left(\tilde{\Psi}_{i}\right)\right\}$, then $\alpha_{0}<0$ and it follows readily from (39) that

$$
\mathbb{E}\left[V\left(\boldsymbol{\epsilon}_{k+1}, k+1, r(k+1)\right) \mid \boldsymbol{\epsilon}_{k}, r(k)=i\right]-V\left(\boldsymbol{\epsilon}_{k}, k, i\right) \leq \alpha_{0}|\boldsymbol{\epsilon}(k)|^{2},
$$

which implies

$$
\mathbb{E}\left[V\left(\boldsymbol{\epsilon}_{k+1}, k+1, r(k+1)\right)\right]-\mathbb{E}\left[V\left(\boldsymbol{\epsilon}_{k}, k, r(k)\right)\right] \leq \alpha_{0}|\boldsymbol{\epsilon}(k)|^{2} .
$$

For an arbitrary positive number $s$, it can be inferred from (40) that

$$
\mathbb{E}\left[V\left(\boldsymbol{\epsilon}_{s+1}, s+1, r(s+1)\right)\right]-\mathbb{E}\left[V\left(\boldsymbol{\epsilon}_{0}, 0, r(0)\right)\right] \leq \alpha_{0} \sum_{k=0}^{s} \mathbb{E}\left[|\boldsymbol{\epsilon}(k)|^{2}\right],
$$

which results in

$$
\sum_{k=0}^{s} \mathbb{E}\left[|\boldsymbol{\epsilon}(k)|^{2}\right] \leq-\frac{1}{\alpha_{0}} \mathbb{E}\left[V\left(\boldsymbol{\epsilon}_{0}, 0, r(0)\right)\right]
$$

It can now be concluded that the series $\sum_{k=0}^{+\infty} \mathbb{E}\left[|\boldsymbol{\epsilon}(k)|^{2}\right]$ is convergent, and therefore

$$
\lim _{k \rightarrow+\infty} \mathbb{E}\left[|\boldsymbol{\epsilon}(k)|^{2}\right]=0 .
$$

This completes the proof of the theorem.

Now we are in a position to deal with the design problem of estimator. The following result is derived easily from Theorem 1, hence its proof is omitted here.

Theorem 2: Let Assumption 1 and condition (5) hold. Then system (6) becomes an asymptotic state estimator of neural network (1) if there exist a set of scalar constants $\vartheta_{i}>0(i \in \mathcal{S})$, a set of matrices $P_{i}>0(i \in \mathcal{S})$, a set of matrices $X_{i}(i \in \mathcal{S})$, two matrices $Q>0$ and $R>0$, and three sets of diagonal matrices $\Omega_{i}>0, \Theta_{i}>0$ and $\Delta_{i}>0(i \in \mathcal{S})$ such that the following LMIs hold:

$$
\Phi_{i} \triangleq\left[\begin{array}{cccccccc}
\Xi_{i} & \Omega_{i} \Lambda_{2} & \Theta_{i} \Sigma_{2} & 0 & \Delta_{i} \Upsilon_{2} & 0 & 0 & \bar{Y}_{i}^{T} \\
\Omega_{i} \Lambda_{2} & -\Omega_{i} & 0 & 0 & 0 & 0 & 0 & A^{T}(i) \bar{P}_{i} \\
\Theta_{i} \Sigma_{2} & 0 & \kappa_{0} Q-\Theta_{i} & 0 & 0 & 0 & 0 & 0 \\
0 & 0 & 0 & -Q & 0 & 0 & 0 & B^{T}(i) \bar{P}_{i} \\
\Delta_{i} \Upsilon_{2} & 0 & 0 & 0 & \kappa_{i} R-\Delta_{i} & 0 & 0 & 0 \\
0 & 0 & 0 & 0 & 0 & \frac{1}{\tau_{2, i}} R & 0 & C^{T}(i) \bar{P}_{i} \\
0 & 0 & 0 & 0 & 0 & 0 & -\vartheta_{i} I & \bar{P}_{i} \\
\bar{Y}_{i} & \bar{P}_{i} A(i) & 0 & \bar{P}_{i} B(i) & 0 & \bar{P}_{i} C(i) & \bar{P}_{i} & -\bar{P}_{i}
\end{array}\right]<0, \quad(i \in \mathcal{S})
$$

where $\bar{Y}_{i}=\bar{P}_{i} D(i)+X_{i} E(i)$, and $\bar{P}_{i}, \Xi_{i}, \kappa_{0}$ and $\kappa_{i}$ are defined as in Theorem 1 . Furthermore, the estimate gain matrix can be taken as $K(i)=\bar{P}_{i}^{-1} X_{i}(i \in \mathcal{S})$. 
Remark 4: In Theorem 1 and Theorem 2, sufficient conditions are provided for the system (6) to be an asymptotic state estimator. Such conditions are expressed in the form of LMIs, which could be easily checked by utilizing the recently developed interior-point methods available in Matlab toolbox, and no turning of parameters will be needed. It should be mentioned that, in the past decade, LMIs have gained much attention for their computational tractability and usefulness in many areas because the so-called interior point method has been proven to be numerically very efficient for solving the LMIs.

\section{A NUMERICAL EXAMPLES}

In this section, we present a simple example to demonstrate the approach addressed.

Example 1: Consider a three-neuron neural network (1) with the following parameters:

$$
\begin{aligned}
& \tau_{1,1}=7, \tau_{1,2}=8, \tau_{2,1}=3, \tau_{2,2}=5, D(1)=\operatorname{diag}\{1.2,0.7,0.8\}, D(2)=\operatorname{diag}\{1.3,0.8,0.6\}, \\
& A(1)=\left[\begin{array}{ccc}
0.2 & -0.5 & 0.1 \\
0.2 & -0.4 & 0 \\
0 & -0.1 & 0.2
\end{array}\right], A(2)=\left[\begin{array}{ccc}
0.6 & -0.2 & 0.1 \\
0.1 & -0.1 & 0.2 \\
0.1 & 0 & 0.2
\end{array}\right], B(1)=\left[\begin{array}{ccc}
0.2 & 0.2 & 0.2 \\
0.2 & -0.2 & 0 \\
0.2 & -0.1 & -0.1
\end{array}\right] \text {, } \\
& B(2)=\left[\begin{array}{ccc}
0.2 & 0.2 & 0.1 \\
0.2 & -0.2 & 0 \\
0.3 & -0.1 & -0.1
\end{array}\right], C(1)=\left[\begin{array}{ccc}
0.2 & 0.2 & -0.1 \\
0 & 0.4 & 0.3 \\
-0.3 & 0 & 0.2
\end{array}\right], C(2)=\left[\begin{array}{ccc}
0.2 & -0.2 & 0.1 \\
0.1 & 0.2 & 0.3 \\
0.8 & 0 & 0.2
\end{array}\right] \text {, } \\
& E(1)=E(2)=\left[\begin{array}{lll}
1 & 0 & 0 \\
0 & 1 & 0
\end{array}\right], \boldsymbol{\Pi}=\left[\begin{array}{ll}
0.6 & 0.4 \\
0.5 & 0.5
\end{array}\right] \text {. }
\end{aligned}
$$

Take the activation functions as follows:

$$
\begin{aligned}
& f_{1}(s)=g_{1}(s)=h_{1}(s)=-\tanh (0.6 s), \quad f_{2}(s)=g_{2}(s)=h_{2}(s)=\tanh (0.2 s), \\
& f_{3}(s)=g_{3}(s)=h_{3}(s)=0.4 \tanh (s) .
\end{aligned}
$$

It is easy to see that $\Lambda_{1}=\Sigma_{1}=\Upsilon_{1}=0, \Lambda_{2}=\Sigma_{2}=\Upsilon_{2}=\operatorname{diag}\{-0.3,0.1,0.2\}$. In addition, letting

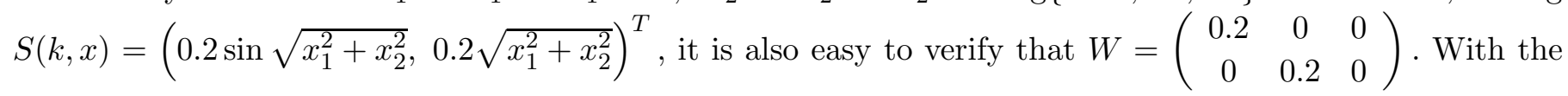
above parameters, by using Matlab LMI Toolbox, we solve the LMIs (41) and obtain the feasible solution as follows:

$$
\begin{aligned}
& P_{1}=\left[\begin{array}{ccc}
3.0831 & -0.3844 & 0.1002 \\
-0.3844 & 2.3970 & -0.7316 \\
0.1002 & -0.7316 & 1.0777
\end{array}\right], P_{2}=\left[\begin{array}{ccc}
4.3869 & -0.2753 & -0.1175 \\
-0.2753 & 2.5613 & -0.6949 \\
-0.1175 & -0.6949 & 0.7936
\end{array}\right] \text {, } \\
& Q=\left[\begin{array}{lll}
1.5023 & 0.5528 & 0.1044 \\
0.5528 & 3.8353 & 0.0235 \\
0.1044 & 0.0235 & 0.3990
\end{array}\right], R=\left[\begin{array}{ccc}
0.0808 & 0.1546 & 0.0013 \\
0.1546 & 0.9912 & -0.0115 \\
0.0013 & -0.0115 & 0.0110
\end{array}\right] \text {, } \\
& X_{1}=\left[\begin{array}{cc}
-4.1167 & 0.3365 \\
0.4422 & -1.1396 \\
0.0671 & -0.1955
\end{array}\right], X_{2}=\left[\begin{array}{cc}
-4.2016 & 0.2623 \\
0.4774 & -1.4941 \\
-0.0706 & -0.1726
\end{array}\right] \text {, } \\
& \Omega_{1}=\operatorname{diag}\{4.0414,16.9336,0.9398\}, \Omega_{2}=\operatorname{diag}\{8.2832,7.5886,1.2245\} \\
& \Theta_{1}=\operatorname{diag}\{4.9918,11.0612,1.2956\}, \Theta_{2}=\operatorname{diag}\{4.9115,10.8292,1.3080\} \text {, } \\
& \Delta_{1}=\operatorname{diag}\{9.3179,22.5366000,1.4407\}, \quad \Delta_{2}=\operatorname{diag}\{20.5649,18.4063,2.4857\} \text {, } \\
& \vartheta_{1}=19.7298, \vartheta_{2}=15.5312 \text {. }
\end{aligned}
$$

From Theorem 2, (6) becomes an asymptotic state estimator of neural network (1) with the given parameters, 
and the estimate gain matrices can be taken as

$$
K(1)=\left[\begin{array}{cc}
-1.1369 & 0.0335 \\
0.0600 & -0.6602 \\
0.1296 & -0.6942
\end{array}\right], K(2)=\left[\begin{array}{cc}
-1.1230 & -0.0060 \\
0.0237 & -0.8410 \\
-0.0678 & -0.8256
\end{array}\right]
$$

\section{Conclusions}

In this paper, we have studied the state estimation problem for a class of discrete-time neural networks with Markovian Parameters and mode-dependent mixed time-delays. An asymptotic state estimator is designed to estimate the neuron states, through available output measurements, such that the dynamics of the estimation error is globally stable in the mean square. By using new Lyapunov-Krasovskii functional, we have established an LMI approach to derive the sufficient conditions guaranteeing the existence of the asymptotic state estimators. The explicit expression of the desired estimator has been parameterized by means of the solution to an LMI. A simulation example has been used to illustrate the usefulness of the derived LMI-based conditions. It should be pointed out that the main results presented in this paper can be extended to stability analysis, filter design and control applications for other discrete-time delayed systems (e.g. genetic regulatory networks), see [22-27] and the references therein.

\section{REFERENCES}

[1] S. Arik, IEEE Transactions on Circuits Systems -I 47(2000)1089.

[2] M. P. Joy, Neural Networks 13(2000)613.

[3] Z. Wang, D. Ho and X. Liu, IEEE Trans. Neural Networks 16(1)(2005)279.

[4] J. Cao, D.-S. Huang and Y. Qu, Chaos, Solitons \& Fractals 23(1)(2005)221.

[5] J. Cao and D. Ho, Chaos, Solitons \& Fractals 24(5)(2005)1317.

[6] H. Huang and J. Cao, Applied Mathematics and Computation 142(1)(2003)143.

[7] V. Singh, Chaos, Solitons \& Fractals 29(2)(2006)470.

[8] Q. Song, Z. Zhao and Y. Li, Physics Letters A 335(2-3)(2005)213.

[9] Q. Song and Z. Zhao, Chaos, Solitons \& Fractals 25(2)(2005)393.

[10] Z. Wang, Y. Liu and X. Liu, Physics Letters A 345(4-6)(2005)299.

[11] H. Zhao, Neural Networks 17(2004)47.

[12] H. Zhao, Physics Letters A 336(2005)331.

[13] Y. Liu, Z. Wang and X. Liu, Neural Networks 19(5)(2006)667.

[14] F. M. Salam and J. Zhang, Adaptive neural observer with forward co-state propagation. In: Proc. International Joint Conference on Neural Networks (IJCNN '01), Washington, U.S.A., Vol.1, 2001, pp.675-680.

[15] R. Habtom and L. Litz, Estimation of unmeasured inputs using recurrent neural networks and the extended Kalman filter. In: Proc. International Conference on Neural Networks, Houston, U.S.A., Vol. 4, 1997, pp.2067-2071.

[16] V. Elanayar and Y. Shin, IEEE Trans. Neural Networks 5(4)(1994)594.

[17] H. Huang, G. Feng and J. Cao, IEEE Trans. Neural Networks 19(8)(2008)1329.

[18] P. Tino, M. Cernansky and L. Benuskova, IEEE Trans. Neural Networks 15(1)(2004)6.

[19] Z. Wang, Y. Liu, L. Yu and X. Liu, Phys. Lett. A 356(4-5)(2006)346.

[20] Y. Liu, Z. Wang and X. Liu, Physics Letters A 372(22)(2008)3986.

[21] Y. Liu, Z. Wang, J. Liang and X. Liu, IEEE Transactions on Systems, Man, and Cybernetics - Part B 38(5)(2008)1314.

[22] Z. Wang, H. Gao, J. Cao and X. Liu, IEEE Trans. NanoBioscience 7(2)(2008)154.

[23] Z. Wang, Y. Liu and X. Liu, Automatica 44(5)(2008)1268.

[24] Z. Wang, F. Yang, D. W. C. Ho, S. Swift, A. Tucker and X. Liu, IEEE Trans. NanoBioscience 7(1)(2008)44.

[25] H. Gao and T. Chen, IEEE Trans. Automatic Control 52(2)(2007)328.

[26] H. Gao and C. Wang, Asian Journal of Control 7(2)(2005)81.

[27] H. Gao and C. Wang, IEEE Trans. Signal Processing 52(6)(2004)1631. 